\title{
手性缩醛的催化不对称合成研究进展
}

\author{
杜鹏 周海峰* 沈冠硕 邹坤 \\ (三峡大学生物与制药学院 天然产物研究与利用湖北省重点实验室 宜昌 443002)
}

\begin{abstract}
摘要 缩醛是许多药物和天然产物分子中常见的结构单元, 研究发现手性缩醛的生物活性通常优于其消旋体. 由于手 性缩醛存在消旋化倾向, 其催化不对称合成具有挑战性, 近年来才引起关注. 综述了目前手性 $N, N$-缩醛、 $N, O$-缩醛、 $N, S$-缩醛、 $O, O$ - 缩醛的催化不对称合成研究进展, 及其在含缩醉骨架手性药物合成中的应用.

关键词 手性缩醛; 催化不对称合成; 手性磷酸
\end{abstract}

\section{Catalytic Asymmetric Synthesis of Chiral Acetals}

\author{
Du, Peng Zhou, Haifeng* Shen, Guanshuo Zou, Kun \\ (Hubei Key Laboratory of Natural Products Research \& Development, College of Biological \& Pharmaceutical Sciences, \\ China Three Gorges University, Yichang 443002)
}

\begin{abstract}
Acetals are common building blocks of many drugs and natural product molecules. It was found that the biological activity of chiral acetals is usually superior to their racemates. The catalytic asymmetric synthesis of chiral acetals is challenge for its racemization and attracts attention until recent years. In this review, the recent studies on catalytic asymmetric synthesis of chiral aminals, $\mathrm{N}, \mathrm{O}$-acetals, $\mathrm{N}, \mathrm{S}$-acetals, $\mathrm{O}, \mathrm{O}$-acetals, as well as its application in synthesis of chiral drugs containing acetal moieties are reviewed.
\end{abstract}

Keywords chiral acetals; catalytic asymmetric synthesis; chiral phosphoric acid

缩醛骨架是许多药物和天然产物分子中常见的结 构单元，如治疗高血压药物喹乙宗(Aquamox)、异丁噻 嗪 (Thiabutazide $)^{[1]}$, 拟副交感神经药西维美林 (Cevimeline) $)^{[2]}$, 治痉挛药哌泊索仑(Pipoxolan)(图 1) ${ }^{[3]}$. 2008 年, Brown 小组研究发现含缩胺骨架的二氢喹唑啉 酮 $(S)$-对映异构体的生物活性优于其 $(R)$-对映异构体 ${ }^{[4]}$. 虽然关于缩醛的反应研究较多 ${ }^{[5]}$, 但是由于手性缩醛化 合物稳定性较差, 存在消旋化倾向, 其催化不对称合成 近年来才引起关注. 在本综述中, 我们对手性 $N, N$-缩 醛、 $N, O$ - 缩醛、 $N, S$ - 缩醛、 $O, O$ - 缩醛的催化不对称合成 研究进展进行概述.

\section{1 手性 $N, N$-缩醛的催化不对称合成}

2005 年, Antilla 等 ${ }^{[6]}$ 率先报道以手性磷酸 $(R)-\mathrm{PA} 1$ 为 催化剂, 通过磺酰胺或者酰亚胺与 $N$-酰基芳香醛亚胺 不对称加成合成手性 $N, N$-缩酫的方法(Eq. 1), 对映选择
性可达到 99\%.

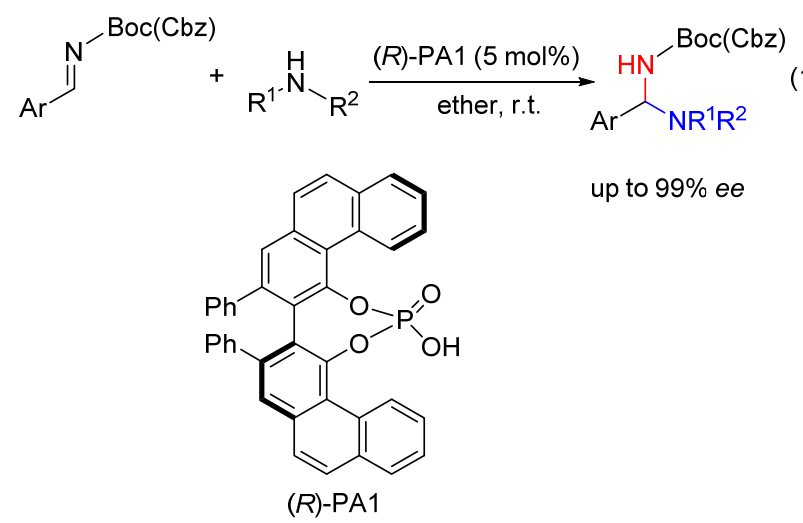

2012 年, Ishihara 小组 ${ }^{[7]}$ 发现在手性双磺酸 $(R)$-SA 与 2,6-二芳基吡啶铵盐的催化下, 酰胺与 $N$-酰基芳香醛亚 胺反应也能顺利得到手性 $N, N$-缩醛, 对映选择性可达 98\% (Eq. 2).

\footnotetext{
* E-mail: haifeng-zhou@hotmail.com
}

Received March 9, 2015; revised March 30, 2015; published online April 10, 2015.

Project supported by the National Natural Science Foundation of China (No. 21202092) and the Startup Foundation from China Three Gorges University (No. KJ2012B080).

国家自然科学青年基金(No. 21202092)和三峡大学人才科研启动基金(No. KJ2012B080)资助项目. 
<smiles>CC[C@H]1NC(=O)c2cc(S(N)(=O)=O)c(Cl)cc2N1</smiles>

Aquamox

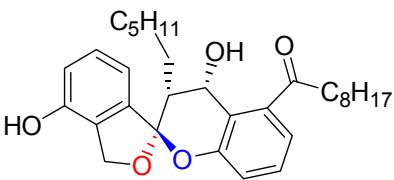

Paecilospirone<smiles>CC(C)C[C@@H]1Nc2cc(Cl)c(S(N)(=O)=O)cc2S(=O)(=O)N1</smiles>

Thiabutazide<smiles>CC1CN2CCC(CC2)CO1</smiles>

Cevimeline<smiles>Cc1ccc(S(=O)(=O)n2c(CN(C(=O)[C@H]3NC(=O)c4ccccc4N3)c3ccccc3)cc3ccccc32)cc1</smiles><smiles>COc1ccc2c(c1)CCN1C(=O)c3ccccc3SC21</smiles>

Sedative<smiles>CC(NC(=O)Cc1ccccc1)C(=O)NC(Nc1ccc(S(N)(=O)=O)cc1)C(=O)OCc1ccccc1</smiles>

E. Coli inhibitor<smiles>O=C1c2cc3c(cc2OC2CCCN12)OCCO3</smiles>

CX-614

an ampakine drug

图 1 含缩醛骨架的药物和天然产物

Figure 1 Drugs and natural products containing acetals

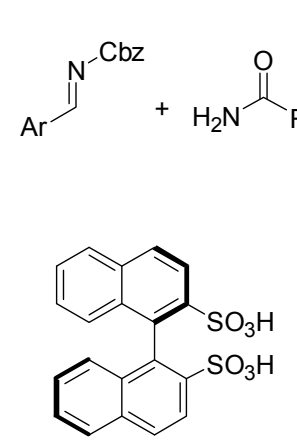

(R)-SA

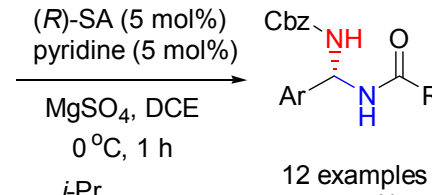<smiles>CCCc1cc(C(C)C)c(-c2cccc(-c3c(P)cc(C(C)C)cc3P(C)C)n2)c(P(C)C)c1</smiles>
up to $98 \%$ ee

2,3-二氢喹唑啉酮化合物具有重要生物活性, 且其 对映异构体活性不同 ${ }^{[4]}$. 直到 2008 年, List 等 $^{[8]}$ 才首次报 道了 2,3-二氢喹唑啉酮的催化不对称合成, 以手性磷酸 $(S)-\mathrm{PA} 2$ 为催化剂, 通过邻氨基苯甲酰胺与脂肪醛在低 温条件下 $\left(-45{ }^{\circ} \mathrm{C}\right)$ 反应 $24 \mathrm{~h}$, 得到手性 2,3-二氢喹唑啉 酮化合物，产率 $72 \%$ ～94\%，对映选择性 $50 \% \sim 98 \%$ (Eq. 3).<smiles>[R1]c1ccc2c(c1)NC([R])NC2=O</smiles>

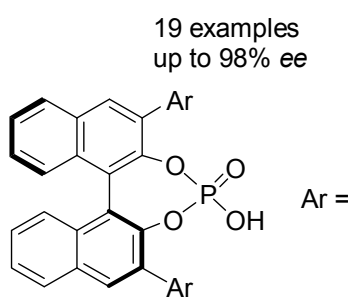

(S)-PA2<smiles>CC(C)c1cc(-c2c3ccccc3cc3ccccc23)cc(C(C)C)c1N</smiles>

与此同时, Rueping 小组 ${ }^{[9]}$ 也报道了具有相同骨架手 性磷酸催化的类似反应，产率 $73 \% \sim 93 \%$ ，对映选择性 $80 \% \sim 92 \%$, 但所使用的醛局限于邻位无取代基的芳香 醛. 林旭峰教授 ${ }^{[10]}$ 以手性螺环骨架的磷酸 $(S)-\mathrm{PA} 3$ 为催 化剂，实现了邻氨基苯甲酰胺与邻位取代的芳香醛反应 制备手性 2,3-二氢喹唑啉酮化合物的方法，对映选择性 可达到 98\% (Eq. 4).
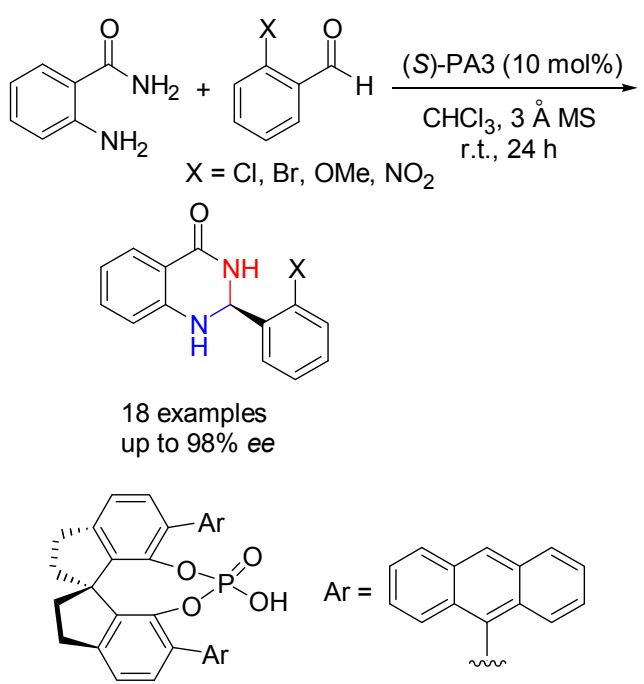

(S)-PA3

2012 年，田士凯教授 ${ }^{[11]}$ 研究发现以手性磷酸 $(R)$ PA4 为催化剂, 邻氨基苯甲酰胺和亚胺为原料也可以合 成 2,3-二氢喹唑啉酮，产率 54\% 90\%，对映选择性 $83 \% \sim 97 \%$ (Eq. 5).

除了手性磷酸有机小分子催化剂外, 2012 年, Kesavan 等 ${ }^{[12]}$ 首次将手性过渡金属配合物 $\mathrm{Sc}(\mathrm{III})-i n d a-$ pybox 应用到 2,3-二氢喹唑啉酮的不对称合成，该催化 体系适用于邻位无取代基的芳香醛和脂肪醛，产率 $80 \% \sim 97 \%$ ，对映选择性 80\% 98\% (Eq. 6). 


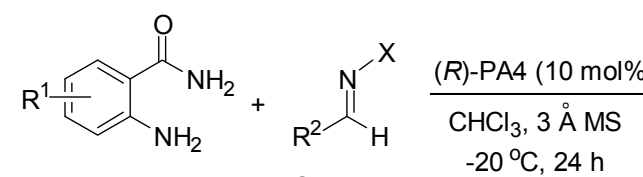<smiles>[R]C1NC(=O)c2cc[R1]cc2N1</smiles>

18 examples up to $97 \%$ ee<smiles>N#[W]c1cc2ccccc2c(OP(=O)(O)O[Na])c1-c1c([Al])cc2ccccc2c1O</smiles><smiles>CCc1c2ccccc2cc2cc(C)ccc12</smiles>

$(R)$-PA4

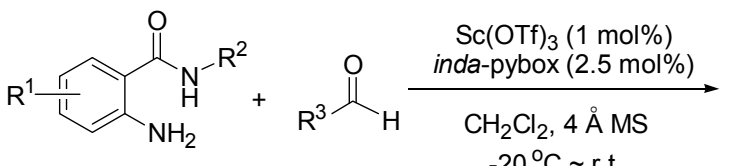<smiles>[R1]c1ccc2c(c1)NC([Y])N([R])C2=O</smiles>
$-20^{\circ} \mathrm{C} \sim$ r.t.

(6)<smiles></smiles>

邻氨基苯甲酰胺和靛红类化合物(酮羰基)在手性磷 酸的催化下, 也可以生成具有重要生物活性的手性螺环 $N, N$-缩醛，产率 $42 \% \sim 82 \%$, 对映选择性 $42 \% \sim 95 \%$ (Eq. 7) ${ }^{[13]}$.

上述方法是在手性催化剂作用下, 经过胺与醛缩 合、酰胺与亚胺不对称加成来合成手性 $N, N$-缩醛. 2013 年, Toste 研究组 ${ }^{[14]}$ 设计并合成了系列 3,3'-三唑取代含 BINOL 骨架的手性磷酸(Triazole-PA), 并成功应用到不 对称交叉脱氢偶联对映选择性生成 $\mathrm{C}-\mathrm{N}$ 键的反应, 得 到含 1,2,3,4-四氢异喹啉骨架的手性环状 $N, N$-缩醛产物, 产率 42\% 93\%, 对映选择性 60\% 94\%, 3,3'三唑取代 基不仅在催化活性位点附近产生空间位阻效应，它与底 物分子间的氢键作用是决定对映选择性高低的关键( Eq. 8). 龚流柱课题组 ${ }^{[15]}$ 以手性布朗斯特酸为催化剂, 通过 邻氨基苯乙醛酸酯与芳香胺缩合、不对称 $[1,5]$-氢转移、 环合反应实现了环状 $N, N$-缩醛的不对称合成, 产率 $32 \% \sim 81 \%$, 对映选择性 72\% 84\% (Scheme 1).

2014 年, 邱立勤课题组 ${ }^{[16]}$ 发展了一种手性磷酸

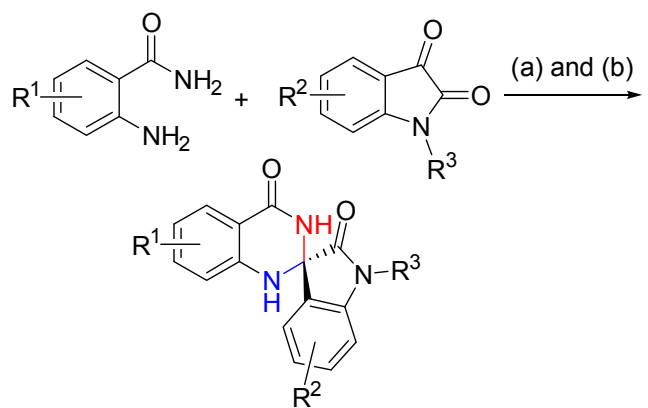

15 examples up to $95 \%$ ee

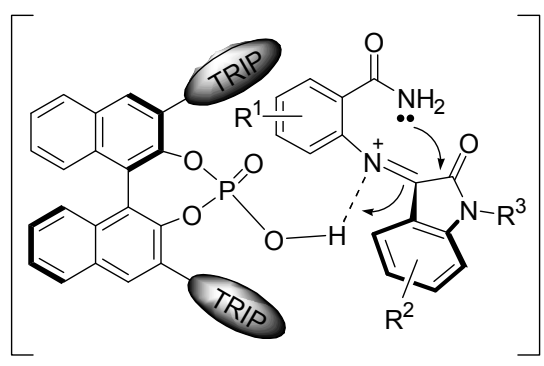

(a) condensation

(b) intramolecular amide addition
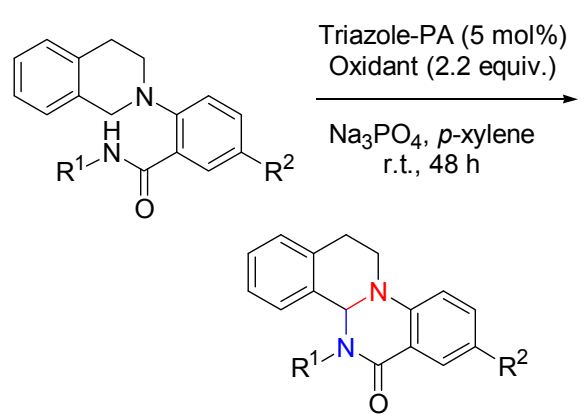

18 examples up to $94 \%$ ee<smiles></smiles><smiles>CC1(C)CC(NC(=O)OCc2ccccc2)CC(C)(C)[N+]1=O</smiles>

(S)-PA5 催化的 2-醛基苯甲酸甲酯与单烷基乙二胺经过 亚胺化反应一亲核加成-内酰胺化 “一锅” 串联反应合成 手性环状 $N, N$-缩醛的方法，产率高达 $94 \%$ ，对映选择性 高达 96\% (Scheme 2).

在金属配合物和手性磷酸的协同催化下，也可以合 成手性 $N, N$-缩醛. Patil 研究组 ${ }^{[17]}$ 以 $\left(\mathrm{PPh}_{3} \mathrm{AuMe} /(S)-\mathrm{PA} 4\right)$ 为催化剂, 2-炔基苯甲醛与邻氨基苯甲酰胺经对映选择 性缩合、氢胺化反应得到含 $1,2-$ 二氢异喹啉骨架的环状 手性 $N, N$-缩醛，产率可达到 95\%，对映选择性可达到 98\% (Scheme 3). 
<smiles>[R]CC([R])C(=O)c1c[R1]([H])ccc1N(C[R])C[R]</smiles><smiles></smiles>

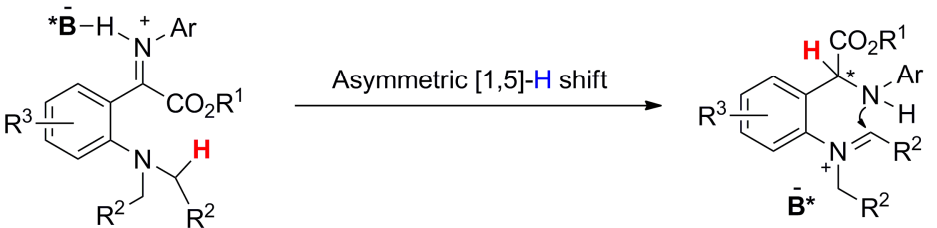

图式 1 经分子内 [1,5]-氢转移合成缩醛

Scheme 1 Synthesis of acetals via intramolecular [1,5]-H shift

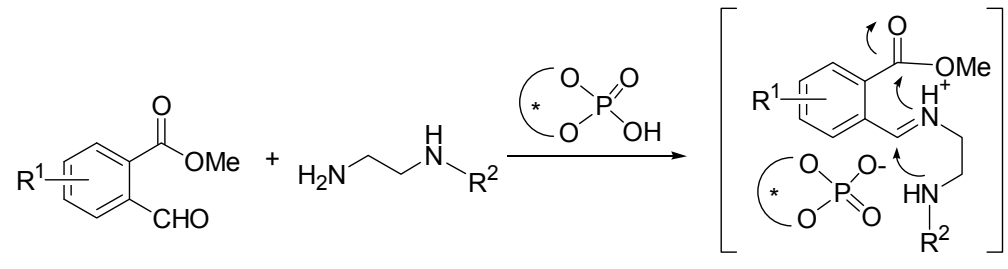

(a) nucleophilic addition

(b) lactamization

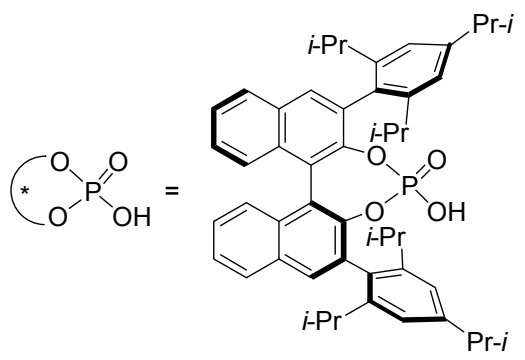

(S)-PA5

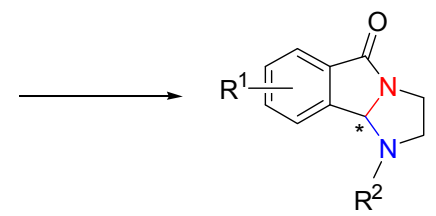

16 examples up to $96 \%$ ee

图式 2 经内酰胺化合成缩醛

Scheme 2 Synthesis of acetals via lactamization

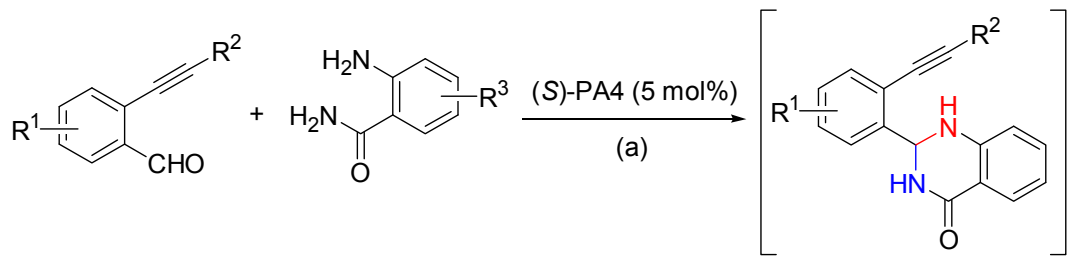

(a) Enantioselective condensation (b) Alkyne hydroamination

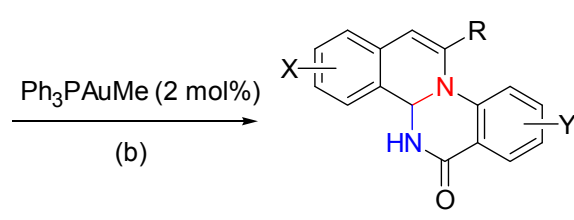

24 examples up to $98 \%$ ee

图式 3 金属络合物和手性磷酸协同催化

Scheme 3 Cooperative catalysis by metal complex and chiral phosphoric acid 
龚流柱课题组 ${ }^{[18]}$ 也报道了在金络合物与手性磷酸 $\left(\mathrm{PPh}_{3} \mathrm{AuNTf}_{2} /(R)-\mathrm{PA} 5\right)$ 协同催化下，1-(2-乙炔苯基)吡咯 与对甲氧基苯胺经分子间氢氨化-分子内 $[1,5]$-氢转移不对称加成串联反应生成手性环状 $N, N$-缩醛的方法, 当 金属催化剂和手性磷酸用量分别为 1.5 和 $200 \mathrm{~mol} \%$ 时, 主产物的对映选择性可以达到 97\% (Eq. 9).

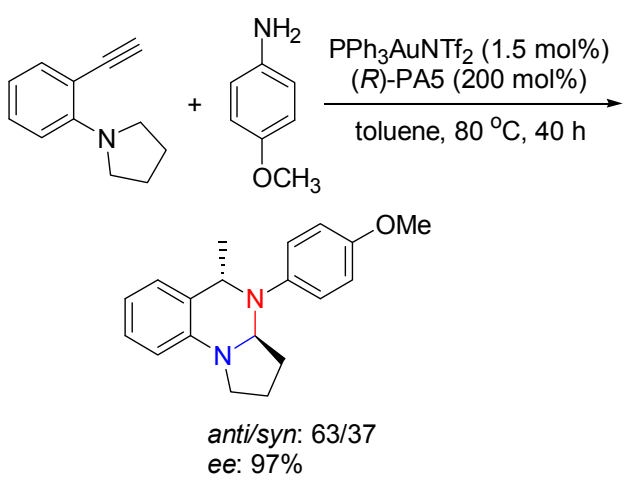

\section{2 手性 N,O-缩醛的催化不对称合成}

2008 年, Antilla 研究组 ${ }^{[19]}$ 以手性磷酸 $(R)-\mathrm{PA} 4$ 为催 化剂, 通过醇与 $N$-酰化亚胺的不对称加成反应得到手性 $N, O$-缩醛, 产率为 $62 \% \sim 99 \%$, 对映选择性为 $65 \%$ 95\% (Eq. 10).

$$
\begin{aligned}
& \mathrm{R}_{\mathrm{R}} \mathrm{R}^{\prime}=\text { aryl, alkyl } \\
& \stackrel{(R)-\mathrm{PA} 4(5 \mathrm{~mol} \%)}{\mathrm{EtOAc} \text {, r.t., } 24 \mathrm{~h}}
\end{aligned}
$$

在含奎宁骨架双官能团有机小分子 $\mathbf{A 1}$ 的催化作用 下, 醇也可以与靛红酮亚胺衍生物发生对映选择性加成 反应得到手性 $N, O$-缩酮, $e e$ 值可达到 78\% (Eq. 11) ${ }^{[20]}$.

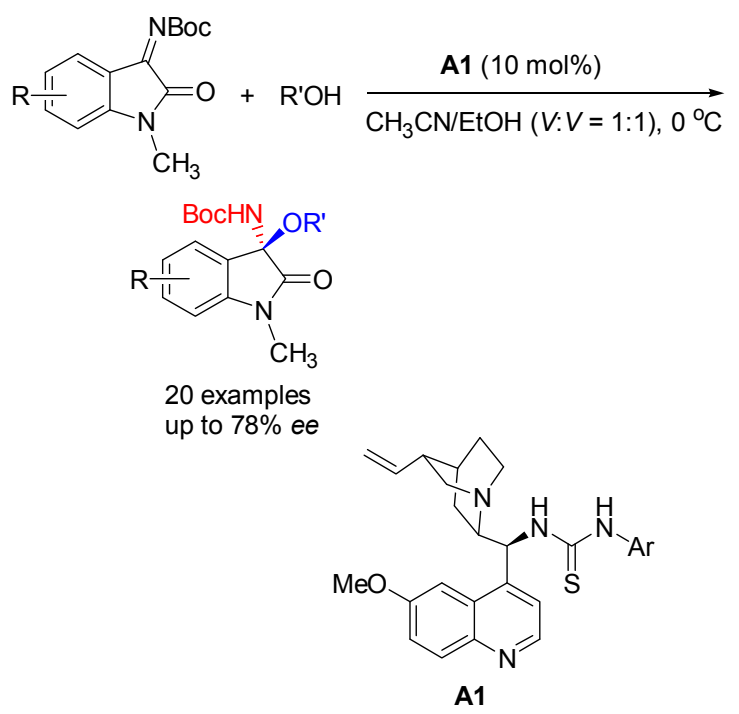

2010 年, List 研究组 ${ }^{[21]}$ 发现在手性磷酰胺 A2 的催化 下，水杨酰胺与脂肪醛(含邻位取代基)经亚胺化反应、 对映选择性加成可以生成环状手性 $N, O$-缩醛，对映选择 性可达到 $97 \%$, 但水杨酰胺与芳香醛的反应，对映选择 性只有 $50 \%$ (Eq. 12).

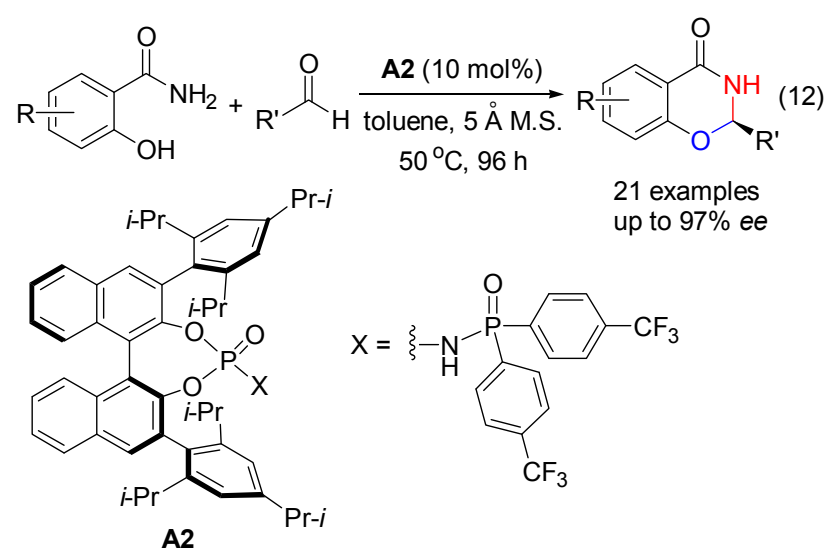

2012 年, Rhee 课题组 ${ }^{[22]}$ 研究发现在手性钯配合物 的催化作用下，高丙炔基磺酰胺与联二烯基烷基醚可以 发生不对称加成反应生成手性 $N, O$-缩醛(Eq. 13).

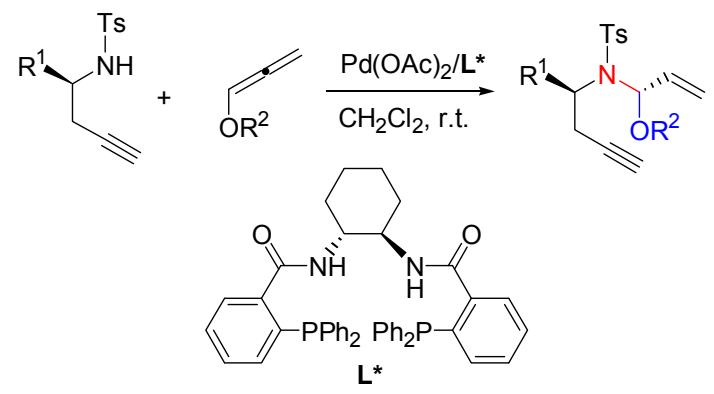

3-烷氧基吲哚-2-酮在含双奎宁骨架有机小分子 A3 的催化下, 可以与偶氮二甲酸二叔丁酯进行不对称加成 反应生成 3,3- $N, O$-取代吲哚-2-酮化合物，产率可达到 $97 \%$, 对映选择性可以达到 $93 \%$ (Eq. 14) ${ }^{[23]}$.
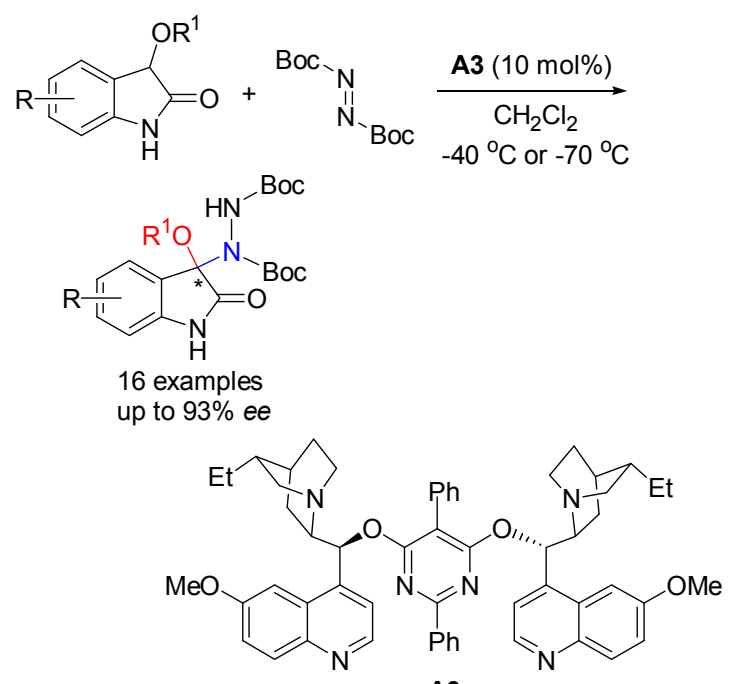

A3 


\section{3 手性 N,S-缩醛的催化不对称合成}

硫醇与亚胺的加成是合成 $N, S$-缩醛最直接的方法, 2011 年, Antilla 研究组 ${ }^{[24]}$ 最先报道了 $N, S$-缩醛的催化不 对称合成, 以手性磷酸 $(R)-\mathrm{PA} 5$ 为催化剂, 通过硫醇与 $N$-酰化亚胺不对称加成来实现, 产率和对映选择性均可 达到 99\% (Eq. 15).

$$
\begin{aligned}
& \text { (R)-PA5 (2 mol\%) } \\
& \mathrm{R}_{2} \mathrm{R}^{\prime}=\text { aryl, alkyl }
\end{aligned}
$$

赵刚课题组 ${ }^{[25]}$ 等研究发现, 以含硫脲-季铵盐双官 能团有机小分子 $\mathbf{A 4}$ 为相转移催化剂, $N$-Boc 亚胺或邻氨 基砜与硫醇反应也可以生成手性 $N, S$-缩醛化合物, 该反 应效率高, 对于克级规模的反应, 只需要 $0.1 \mathrm{~mol} \%$ 的催 化剂, 在一 $30{ }^{\circ} \mathrm{C}$ 反应 $5 \mathrm{~min}$, 产率可以达到 $99 \%$, 对映 选择性为 $93 \%$ (Eq. 16).

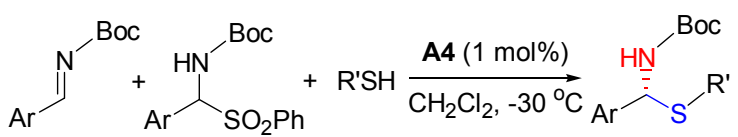

$$
\begin{aligned}
& \text { (N4) }
\end{aligned}
$$

在含奎宁骨架有机小分子 $\mathbf{A 5}$ 的催化下, 3-硫代吲 哚-2-酮与偶氮二甲酸二叔丁酯进行不对称加成可以生 成 3,3-N,S-取代的吲哚-2-酮化合物, 产率可达 98\%, 对 映选择性可达到 $94 \%$ (Eq. 17) ${ }^{[23]}$.
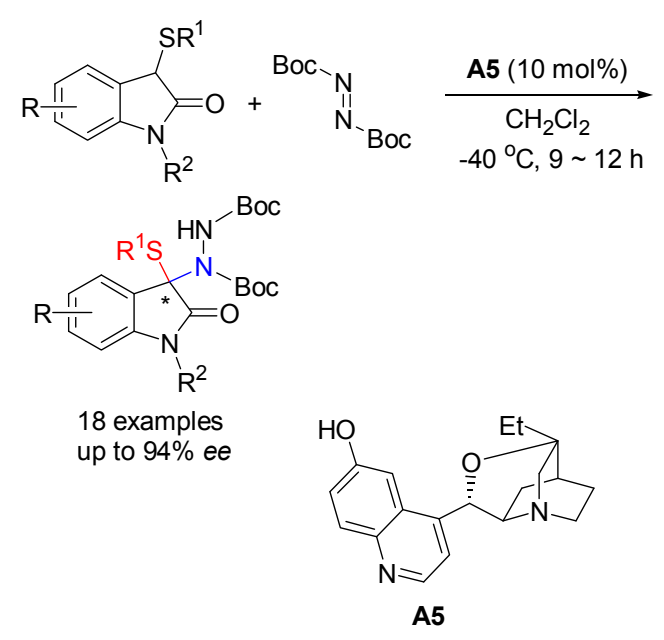
催化的 1,1'-双(2-羟基亚甲基芳基)酮经不对称氢化和缩 酮化反应合成手性螺环 $O, O$-缩酮的方法, 对映选择性 高达 $99 \%$, 并将该方法成功应用到含手性螺环 $O, O$ - 缩酮 骨架双膦配体 $\mathbf{A 9}$ 的制备, 所得手性膦配体对映选择性 可以达到 99\% (Eq. 21).

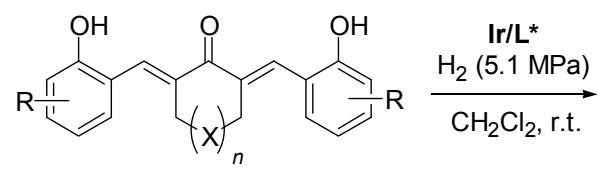<smiles>[Y14]CC1Cc2ccc([R])cc2OC12Cc1ccc([R])cc1O2</smiles>

18 examples up to $>98 / 2$ d.r., $>99 \%$ ee<smiles>C[C+](Br)c1ccccc1</smiles>

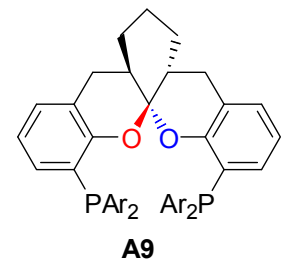

List 研究组 ${ }^{[30 a]}$ 在手性螺环 $O, O$-缩酮的不对称合成 方面取得突破性进展, 他们设计合成了手性磷酰亚胺有 机小分子催化剂 A10, 该催化剂的活性位点具有独特的 手性微环境, 羊弪烷基烯醇醚在其催化作用下发生分子内 不对称加成反应生成手性螺环 $O, O$-缩酮, 对映选择性 可以高达 $97 \%$, 该研究成果发表在国际权威杂志 Nature 上(Eq. 22).<smiles>OCCCCC1=CCCCO1</smiles>

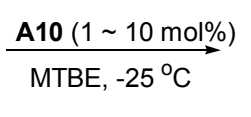

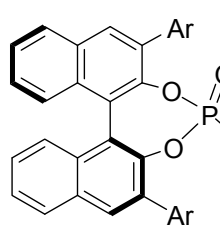<smiles>O=[PH](Oc1cc2ccccc2cc1Cl)Oc1c(Cl)cc2ccccc2c1OP(=O)(O)O</smiles><smiles>CCc1cc(CC)c(N=[W])c(CC)c1</smiles>

该催化剂体系也适用于醛与醇分子间的不对称缩 醛化反应, 如 A10'可以催化醛与邻二醇或 1,3-二醇的不 对称缩醛化反应得到手性 $O, O$-缩醛, 产率可以达到 $93 \%$, 对映选择性可以达到 $99 \%$ (Eq. 23) ${ }^{[30 b]}$.

$$
\begin{aligned}
& \begin{array}{l}
\because \mathrm{R}^{2} \mathrm{OH} \\
\because \mathrm{R}^{1} \mathrm{OH}
\end{array} \\
& \text { r.t., } 2 \mathrm{~d} \\
& 93 \% \text { yield, } 91 \% \text { ee }
\end{aligned}
$$

随后, Nagorny 小组 ${ }^{[31]}$ 研究发现手性磷酸 PA5 也可 以催化羟烷基烯醇醚分子内的不对称加成反应生成手 性螺环 $O, O$ - 缩酮, 对映选择性可以达到 $96 \%$ (Eq. 24).

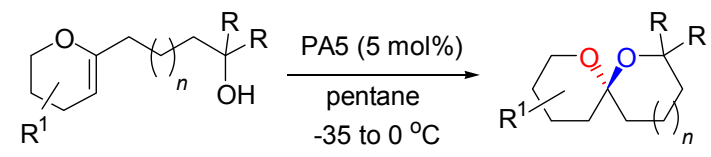

2013 年, 龚流柱课题组 ${ }^{[32]}$ 研究发现 $\mathrm{PPh}_{3} \mathrm{AuMe}$ 和手 性磷酸 $(R)$-PA6 协同催化的炔醇、芳香胺、水杨醛三组 分反应合成手性螺环 $O, O$-缩酮的新方法, 产率可以达 到 97\%，对映选择性可以达到 95\% (Eq. 25). Rodríguez 小 组 ${ }^{[33]}$ 也报道了类似的合成方法, 以(JohnPhos)AuMe 和 $(R)$-PA4 为催化剂, 炔醇、芳香胺、乙醛酸通过三组 份 “一锅” 反应得到手性螺环 $O, O$ - 缩酮, 对映选择性可 以达到 96\% (Eq. 26).
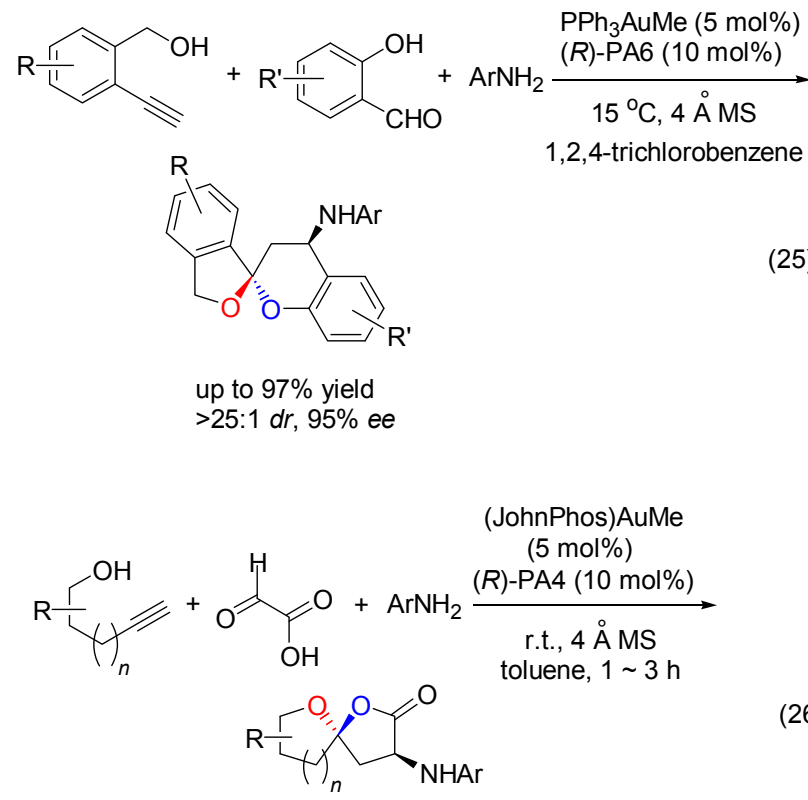

\section{5 手性缩醛催化不对称合成在手性药物合成中 的应用}

研究发现 2,3-二氢喹唑啉酮类药物 $(S)$-对映异构体 
的生物活性优于 $(R)$-对映异构体 ${ }^{[4]}$. Guidotti 研究组 ${ }^{[34]}$ 通 过手性拆分的方法得到噻嗪类化合物的单一对映异构 体, 并进行了动物实验. 研究结果表明右旋对映异构体 的活性高于左旋对映异构体. 由于缩胺的催化不对称合 成近几年才得以实现, 目前市售的 2,3-二氢喹唑啉酮和 噻嗪类药物均为消旋体, 如治疗高血压药喹乙宗 (Aquamox)、美托拉宗 (Metolazone) 、环戊噻嗪 (Cyclopenthiazide)、贝美噻嗪(Bemetizide)、氟芐噻嗪 (Bendroflumethiazide)等. 近年来, 随着手性 $N, N$-缩醛不 对称合成技术的发展, 研究者也对 2,3-二氢喹唑啉酮和 噻嗪类药物的不对称合成进行了初步研究. List 研究 组 ${ }^{[8]}$ 将其发展的手性 $N, N$-缩醛的合成方法应用到喹乙宗 和几种噻嗪类降压药的不对称合成, 以手性磷酸 $\mathrm{A11}$ 为 催化剂, 邻氨基苯磺酰胺或邻氨基苯甲酸与相应的醛在 室温下反应 $7 \mathrm{~d}$, 其中噻嗪类化合物对映选择性可以达 到 90\%以上，喹乙宗的对映选择性可以达到 $61 \%$ (图 2).

抗血栓剂 M55529 含 $N, O$-缩醛手性中心, 其左旋异 构体的 Factor Xa 抑制活性是右旋异构体的 65 倍. Mi- kami 研究组 ${ }^{[35]}$ 报道了手性金属络合物(Salen-Mn)催化的 M55529 的不对称合成，对映选择性可以达到 $86 \%$ (Scheme 4).

\section{6 结论}

本文对已报道的手性 $N, N$-缩醛、 $N, O$-缩醛、 $N, S$-缩 醛、 $O, O$-缩醛的催化不对称合成方法进行了简要的综述, 已报道的合成策略主要是通过手性磷酸有机小分子催 化的酰胺、醇或硫醇与亚胺的不对称加成反应来实现， 虽然取得一些重要研究成果，但是策略较单一，底物适 应范围有限，很多具有重要生物活性的缩醛类化合物的 不对称合成尚未实现. 即使目前研究较多的 2,3-二氢喹 唑啉酮的不对称合成，所报道的方法都是以邻氨基苯甲 酰胺与醛为原料，多数情况下只适合芳香醛，脂肪醛的 反应需在超低温下进行，醛基的邻位还需带有取代基. 手性缩醛的催化不对称合成研究还拥有广阔的空间，发 展适用范围更广、更高效、更温和的催化不对称合成<smiles>CC(C)C[C@@H]1Nc2cc(Cl)c(S(N)(=O)=O)cc2S(=O)(=O)N1</smiles>

$(R)$-Thiabutazide $81 \%$ yield, $91 \%$ ee<smiles>NS(=O)(=O)c1cc2c(cc1C(F)(F)F)N[C@@H](Cc1ccccc1)NS2(=O)=O</smiles>

(R)-Bendroflumethiazide $80 \%$ yield, $92 \%$ ee<smiles>NS(=O)(=O)c1cc2c(cc1Cl)N[C@H](CC1CCCC1)NS2(=O)=O</smiles>

(R)-Cyclopenthiazide $72 \%$ yield, $91 \%$ ee<smiles>CCCCC[C@@H]1Nc2cc(C(F)(F)F)c(S(N)(=O)=O)cc2S(=O)(=O)N1</smiles>

(R)-Penflutizide $74 \%$ yield, $90 \%$ ee

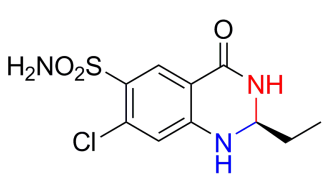

(S)-Aquamox $78 \%$ yield, $61 \%$ ee

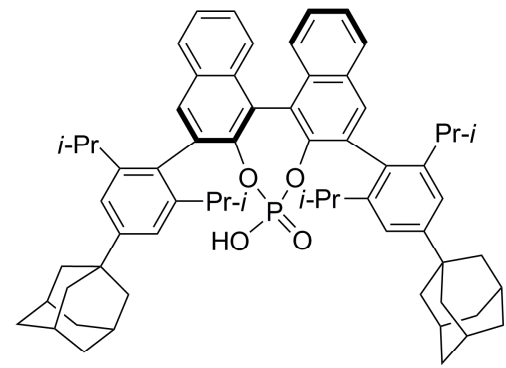

A11

图 2 含手性 $N, N$-缩醛骨架抗高血压药物

Figure 2 Antihypertensive pharmaceuticals containing chiral aminals

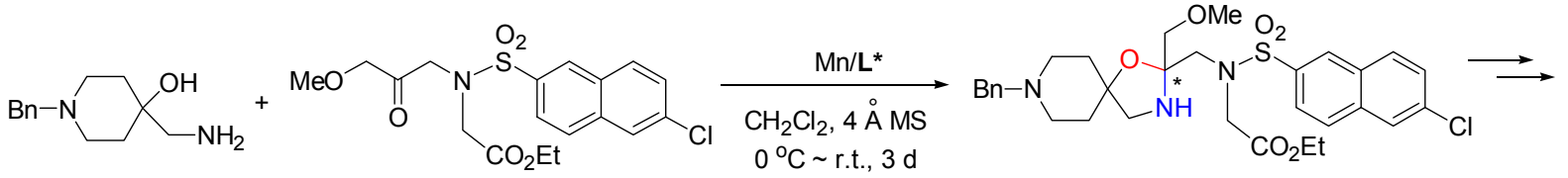<smiles>COCC12CN(S(=O)c3ccc4cc(Cl)ccc4c3)CC(=O)N1CC1(CCN(c3ccncc3)CC1)O2</smiles>

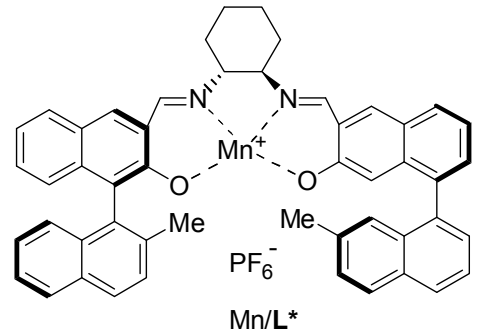

图 4 抗血栓剂 M55529 的不对称合成

Scheme 4 Asymmetric synthesis of antithrombotic M55529 
手性缩醛化合物的新方法, 并成功应用到含缩醛骨架手 性药物的合成是该领域今后研究的重点. 本课题组正在 进行通过芳香杂环化合物催化不对称氢化, 以及 $\mathrm{sp}^{3}$ $\mathrm{C}-\mathrm{H}$ 键活化/不对称交叉脱氢碳-杂键生成等策略来合 成手性缩醛的研究.

\section{References}

[1] Verdel, B. M.; Souverein, P. C.; Egberts, A. C. G.; Leufkens, H. G. M. Ann. Pharm. 2006, 40, 1040.

[2] Mavragani, C. P.; Moutsopoulos, H. M. Clin. Rev. Allergy Immunol. 2007, 32, 287.

[3] Felix, W.; Rimbach, G.; Wengenroth, H. Arzneim. Forsch. 1969, 19, 1860.

[4] Chinigo, G. M.; Paige, M.; Grindrod, S.; Hamel, E.; Dakshanamurthy, S.; Chruszcz, M.; Minor, W.; Brown, M. L. J. Med. Chem. 2008, 51, 4620 .

[5] (a) Liu, N.; Yang, L.; Yang, B.; Wang, J.; Chen. X.; Wu, Q. Chin. J. Org. Chem. 2014, 34, 2523 (in Chinese). (刘宁宁，杨玲，杨蓓，王金棒，陈雪，武钦佩，有机化学，2014, 34, 2523.)

(b) Wang, C.; Li, Y.; Jia, G.; Lu, S.; Liu, Y.; Li, C. Acta Chim. Sinica 2013, 71, 36 (in Chinese).

(王长号, 李英豪, 贾国卿, 卢胜梅, 刘李灿, 化学学报, 2013, 71, 36.)

(c) Chen, J.; Liu, Q.; Dai, X.; Nie, L.; Fang, H.; Wu, X. Chin. J. Org. Chem. 2013, 33, 1 (in Chinese).

(陈杰, 刘钦, 戴小者, 聂凛凛, 房辉辉, 吴小余, 有机化学, 2013, 33, 1.)

(d) Feng, T.; Si, C.; Liu, R.; Fan, X.; Wei, B. Chin. J. Org. Chem. 2013, 33, 1291 (in Chinese).

(冯涛, 司长梅, 刘如成, 范翔, 魏邦国, 有机化学, 2013, 33, 1291.)

(e) Liu, N.; Chen, X.; Zhang, Q.; Wu, Q. Chin. J. Org. Chem. 2014, 34, 1364 (in Chinese).

(刘宁宁，陈䂀，张青山，武钦佩，有机化学，2014，34，1364.)

[6] (a) Rowland, G. B.; Zhang, H.; Rowland, E. B.; Chennamadhavuni, S.; Wang, Y.; Antilla, J. C. J. Am. Chem. Soc. 2005, 127, 15696.

(b) Liang, Y.; Rowland, E. B.; Rowland, G. B.; Perman, J. A.; Anitlla, J. C. Chem. Commun. 2007, 43, 4477.

[7] Hatano, M.; Ozaki,T.; Sugiura, Y.; Ishihara, K. Chem. Commun. 2012, $48,4986$.

[8] Cheng, X.; Vellalath, S.; Goddard, R.; List, B. J. Am. Chem. Soc. 2008, 130, 15786.

[9] Rueping, M.; Antonchick, A. P.; Sugiono, E.; Grenader, K. Angew. Chem., Int. Ed. 2009, 48, 908.

[10] Huang, D.; Li, X.; Xu, F.; Li, L.; Lin, X. ACS Catal. 2013, 3, 2244.

[11] Cheng, D. J.; Tian, Y.; Tian, S. K. Adv. Synth. Catal. 2012, 354, 995.

[12] (a) Prakash, M.; Kesavan, V. Org. Lett. 2012, 14, 1896. (b) Prakash, M.; Jayakumar, S.; Kesavan, V. Synthesis 2013, 2265.

[13] Jiang, Y.; Liu, Y.; Tu, S. J.; Shi, F. Tetrahedron: Asymmetry 2013, $24,1286$.

[14] Neel, A. J.; Hehn, J. P.; Tripet, P. F.; Toste, F. D. J. Am. Chem. Soc. 2013, 135, 14044.

[15] He, Y. P.; Du, Y. L.; Luo, S. W.; Gong, L. Z. Tetrahedron Lett. 2011, 52, 7064.

[16] He, Y.; Cheng, C.; Chen, B.; Duan, K.; Zhuang, Y.; Yuan, B.; Zhang, M.; Zhou, Y.; Zhou, Z.; Su, Y. J.; Cao, R.; Qiu, L. Org. Lett. 2014, 16, 6366.

[17] Patil, N. T.; Mutyala, A. K.; Konala, A.; Tella, R. B. Chem. Commun. 2012, 48, 3094.

[18] (a) He, Y. P.; Wu, H.; Chen, D. F.; Yu, J.; Gong, L. Z. Chem. Eur. J. 2013, 19, 5232 .

(b) Chen, D. F.; Han, Z. Y.; Zhou, X. L.; Gong, L. Z. Acc. Chem. Res. 2014, 47, 2365.

[19] Li, G.; Fronczek, F. R.; Antilla, J. C. J. Am. Chem. Soc. 2008, 130, 12216.

[20] Li, T. Z.; Wang, X. B.; Sha, F.; Wu, X. Y. Tetrahedron 2013, 69, 7314.

[21] Vellalath, S.; Coric, I.; List, B. Angew. Chem., Int. Ed. 2010, 49 , 9749.

[22] Kim, H.; Rhee, Y. H. J. Am. Chem. Soc. 2012, 134, 4011.

[23] Zhou, F.; Zeng, X. P.; Wang, C.; Zhao, X. L.; Zhou, J. Chem. Commun. 2013, 49, 2022.

[24] Ingle, G. K.; Mormino, M. G.; Wojtas, L.; Antilla, J. C. Org. Lett. 2011, 13, 4822.

[25] Wang, H. Y.; Zhang, J. X.; Cao, D. D.; Zhao, G. ACS Catal. 2013, $3,2218$.

[26] Fang, X.; Li, Q. H.; Tao, H.Y.; Wang, C. J. Adv. Synth. Catal. 2013, $355,327$.

[27] Qian, H.; Sun, J. Asian J. Org. Chem. 2014, 3, 387.

[28] Nakamura, S.; Takahashi, S.; Nakane, D.; Masuda, H. Org. Lett. 2015, 17, 106.

[29] (a) Wang, X.; Han, Z.; Wang, Z.; Ding, K. Angew. Chem., Int. Ed. 2012, 51, 936 .

(b) Wang, X.; Guo, P.; Wang, X.; Wang, Z.; Ding, K. Adv. Synth. Catal. 2013, 355, 2900.

[30] (a) Coric, I.; List, B. Nature 2012, 483, 315.

(b) Kim, J. H.; Coric, I.; Vellalath, S.; List, B. Angew. Chem., Int. Ed. 2013, 52, 4474.

[31] Sun, Z.; Winschel, G. A.; Borovika, A.; Nagorny, P. J. Am. Chem. Soc. 2012, 134, 8074

[32] Wu, H.; He, Y. P.; Gong, L. Z. Org. Lett. 2013, 15, 460.

[33] Cala L.; Mendoza, A.; Fananas, F. J.; Rodríguez, F. Chem. Commun. 2013, 49, 2715.

[34] Uzunov, D. P.; Zivkovich, I; Pirkle, W. H.; Costa, E.; Guidotti, A J. Pharm. Sci. 1995, 84, 937.

[35] Saitoh, F.; Nishida, H.; Mukaihira, T.; Aikawa, K.; Mikami, K. Eur. J. Org. Chem. 2006, 10, 2269. 